\title{
9/11: WIE POLITISCH KANN MUSIK SEIN?
}

\author{
Helmut Rösing
}

\section{Bestandsaufnahme}

Zuerst ein kurzer Rückblick. Was geschah am 11. September 2001?

Eine entführte Boeing 767 der American Airlines dringt in den Luftraum über New York ein. Sie nimmt Kurs auf das World Trade Center, in dem bereits Tausende an ihren Schreibtischen sitzen und arbeiten. Um 8.48 Uhr rammt das Flugzeug die Fassade des nördlichen Turms in 300 Metern Höhe. Eine gewaltige Explosion erschüttert die Stadt. Überlebende versuchen sich über die Notausgänge zu retten, es entsteht Panik. Die Katastrophe verdoppelt sich: Um 9.06 Uhr fliegt ein zweiter Jet in den südlichen Turm. Genau 57 Minuten später sackt der südliche Tower nach mehreren Detonationen in sich zusammen, um 10.30 Uhr dann auch der zweite, nördliche Turm. Eine weit über hundert Meter hohe Rauchwolke bedeckt ganz Manhattan. Kurze Zeit später stürzt ein drittes Flugzeug in Washington ins Pentagon und ein viertes zerschellt auf einem Feld bei Pittsburgh.

Viele tausend Menschen wurden Opfer dieses Al-Kaida-Terroranschlags, der die US-amerikanische Nation mitten ins Herz traf: Das Pentagon war und ist die Schaltzentrale der militärischen Macht, und das World Trade Center galt als weithin sichtbares Symbol für ihre ökonomische Kraft und Stärke. In seinen 110 Stockwerken residierten Hunderte von renommierten Firmen aus aller Welt.

Millionen Menschen auf der ganzen Welt verfolgten das Horrorszenario des Anschlags am Bildschirm wie eine Reality-Show. Doch diesmal handelte es sich nicht um eine mediale Inszenierung aus Hollywood, sondern um blutigen Ernst. US-Präsident Bush sprach von einer »nationalen Tragödie«, Bundeskanzler Gerhard Schröder bezeichnete den Anschlag als »Kriegserklärung gegen die gesamte zivilisierte Welt « und bei den Kommentatoren herrschte Einigkeit: Nichts wird mehr so sein, wie es war (alle Zitate: Bild v. 12. September 2001, S. 1). 


\section{Popmusikalische Reaktionen}

Die Musikerinnen und Musiker der populären Musikszenen reagierten schnell. Live-Musikkampagnen fanden statt, Benefizkonzerte für Opfer, Überlebende und Helfer wurden veranstaltet und auf $C D$ erschien bereits im Oktober eine Vielzahl von Titeln, die sich mit dem Ereignis befassten: teilweise eher allgemein, teilweise mit expliziten für bzw. gegen einen Krieg ausgerichteten Botschaften. Das umfangreiche klingende Material, das mir dankenswerter Weise von Thomas Phleps zur Verfügung gestellt wurde, führt quer durch verschiedene Stile (Pop, Spaß-Pop, Folk-Rock, Rhythm and Blues, Soul und HipHop), quer durch verschiedene Präsentations- und Produktionsformen (Benefiz-Live-Veranstaltung, CD, Video, Internetveröffentlichung bzw. MP3File) und quer durch verschiedene Botschaften wie z.B. Artikulation von Entsetzen, Aufruf zum gemeinsamen Gebet, Plädoyer für den Frieden, Aufforderung zum Krieg gegen Terror.

Vor allem die Texte als integraler Bestandteil der Songs steuern durchaus einiges zum konkreten Anlass bei, ohne dass deswegen allzu viele Worte verloren werden müssten. In der Regel reichen bereits einige Schlüsselbegriffe, um beim Hörer die medial vermittelten Bilder des Terroranschlags in Erinnerung zu rufen und überwiegend emotional gefärbte Assoziationsketten zum Geschehen und seinen politischen wie gesellschaftlichen Implikationen in Gang zu setzen: "September day", »that tuesday", "lost towers «, "shots in the summer day«, »fire in the city«, »apocalypse«, »world war 3«. Am Rande sei vermerkt, dass in den Pop-, Country- und Folkrock-Titeln die Texte in der Regel sehr vage und hochgradig metaphorisch angelegt sind, während sich demgegenüber die wortgewaltigen HipHop-Texte meist viel konkreter auf das reale Geschehen beziehen.

Wie aber verhält es sich mit der Musik? Lässt sich auch hier eine konkrete Bezugnahme auf das Geschehen am 11. September ausmachen?

Da gibt es - je nach Musikstil - emphatisch-melodiöse Abschnitte in gemäßigtem Tempo, möglichst zum Mitsingen und Mitsummen; da herrscht dichter Sound mit so manchem Streicherpathos zu mehr oder weniger markanten Bläsereinschüben und wohlgetuneten Gitarrensoli; da gibt es Crescendo-Walzen über mehrere Strophen hinweg oder aber knackige Doublebass-Grundrhythmen zu einigen wenigen prägnanten Electronicsounds und zu coolem Rapper-Parlando; da hört man puristische Unplugged-Musik im Country-Stil, prayerartige Call and Response-Motive mit soul- oder gospelnahen Auszierungen; da wird zitiert, gesampelt, parodiert, persifliert kurz: es findet sich popmusikalisch alles das in den Songs, was man ohnehin 
und je nachdem bei populärer Musik zu erwarten pflegt. Die Suche nach dem Besonderen, Einmaligen, der politischen Brisanz Angemessenen, etwa nach tonmalerisch sprechenden Sounds, nach zerbrechenden bzw. zerbrochenen musikalischen Formen, nach dem, was anders ist, als es war, weil nach dem 11. September angeblich »nichts mehr so sein wird, wie es war « - diese Suche erweist sich als wenig ergiebig. Eine Ausnahme machen allenfalls Masterminds mit dem Song "September In New York«. In RapReviews verweist Damon Brown (2002) diesbezüglich mit Recht auf einen »vicious«, also ebenso fehlerhaften wie bösartigen »drum and bass-beat«, der das rhythmisch-formale Gefüge eindringlich und hautnah ins Wanken bringt. Doch selbst hier noch bleibt offen, inwieweit ein derart musikalisch vermitteltes Bild von möglicherweise einstürzenden Gebäudeteilen für mehr einsteht als eben dieses Bild, das ein Hörbild für sehr verschiedene reale oder auch innermenschlich-psychische Vorgänge sein kann.

Sogar das Verfahren der musikalischen Parodie, die Umtextierung des von Harry Belafonte popularisierten »Banana Boat Song « durch die Crew der Rock \& Roll Morning Show des Senders KOMP, hinterlässt einen seltsam zwiespältigen Eindruck. Der Song changiert zwischen Ernst, Witz, Sarkasmus und überlässt somit dem rezipierenden Subjekt einen erheblichen Interpretationsspielraum.

Die Auswertung der vielen Pop- und Rockmusikprodukte, die explizit eine Reaktion auf den 11. September 2001 darstellen, führt somit zwangsläufig zu grundsätzlichen, die Kommunikationsfähigkeit von Musik betreffenden Fragen: Kann Musik der konkreten Situation eines Terrorszenarios wie dem vom 11. September überhaupt unmissverständlich Ausdruck verleihen? Gibt sie gar etwas davon direkt wieder? Und werden die Sounds zudem mit politischen Botschaften verknüpft, gleichsam als Kommentar zum Geschehen? Was heißt überhaupt »politische Musik«? Kann Musik politisch sein? Verfügt sie über eine hinreichende begriffliche Konkretheit, um auf ein einmaliges Ereignis hinzuweisen und eine klare politische Aussage zu treffen?

\section{Politische Musik - Ansätze zur Begriffsklärung}

Fragen zu stellen ist leider leichter, als sie zu beantworten. Und Zweifel sind gerade auch im wissenschaftlichen Diskurs immer angebracht. Kann Musik politische Kraft entfalten, gar politisch wirksam sein? Sind nicht gerade die Nicht-Diskursivität und das große Transzendenzpotential von Musik Faktoren, die die Musik aus den Niederungen konkreter politischer 
Verhältnisse emporheben und sie gerade darum dazu befähigen, ebenso visionäre wie emotional-abstrakte Gegenentwürfe zur soziopolitischen Alltagswelt zu entwerfen? Müsste dann nicht Musik, wann immer sie erklingt, als politisch im höchst allgemeinen Sinn bezeichnet werden - eben ohne jegliche realpolitische Konkretion? Oder steht - ganz im Gegenteil - das musikalische Transzendenzpotential für das Unpolitische von Musik ein? Wie dem auch sei (und der Fragenkatalog ließe sich ohnehin mühelos weiter fortsetzen): Der Begriff »politische Musik« hat sich in der letzten Zeit derart etabliert, dass ihm im Sachteil der neuen MGG-Enzyklopädie nicht weniger als 20 Spalten gewidmet sind. Nachdem der Begriff zunächst für Musik im Umfeld linksorientierter und gesellschaftskritischer Kreise verwendet wurde (Kampf- bzw. Revolutionsmusik, Musik gegen Diktaturen, Musik in Verbindung mit Anti-Kriegs-Protestbewegungen), hat mittlerweile eine Bedeutungsöffnung stattgefunden: Wo immer mit Musik die »Abweichung vom bürgerlichen Normalzustand « - rechts wie links, oben wie unten - propagiert wird, handelt es sich um politische Musik (Heister 1997: Sp. 1662). Dabei wird das Politische als »eine konzentrierte Form des Gesellschaftlichen « bezeichnet (ebd.: Sp. 1663). Ziel von politischer Musik sei eine wie auch immer geartete "gesellschaftliche Veränderung " (Flammer 1981: 66). Sie widersetze sich damit der Rechtfertigung einer bestehenden gesellschaftlichen Daseinsform (Phleps 1993: 97).

Politische Musik sollte sich, wie autonome Musik auch, nach Ansicht von Otto Kolleritsch avancierter musikalischer Stilmittel bedienen. Ihr progressives Element finde sein Äquivalent im fortschrittlichen kompositorischen Standard. Rückständige musikalische Sprache schwäche den kritischen Gestus und erzeuge regressives Hören (Kolleritsch 1972: 94). Selbst fortschrittliche Texte, so Heiner Goebbels (1980), könnten durch »verdummende Musik « noch paralysiert werden. Gegen derartige von Theodor W. Adornos Musikverständnis und seinen Qualitätskriterien (Adorno 1962) nachhaltig geprägte Auffassungen konterte Tibor Kneif (1977): Das alles könne nur für einen kleinen Kreis von Kennern und Experten gelten. Politische Musik müsse, wie schon die Musik im Gefolge der französischen Revolution gezeigt habe, möglichst plakativ sein, um breite Hörerkreise zu erfassen und ihr Ziel zu erreichen.

Um diesen die musikästhetische Seite betreffenden Streitigkeiten ihre Schärfe zu nehmen, meinte Hansjörg Pauli (1981: 387) kurzerhand: Nicht der musikalische Text, sondern der außermusikalische Kontext »bestimme ganz wesentlich den Sinn eines Werks «. Musik auf sich allein gestellt könne keine eindeutige Stellung beziehen. Das zeige selbst die Geschichte der Vokalmusik. Die Umfunktionierung durch Kontrafaktur habe nicht einmal vor 
dem »Horst-Wessel-Lied« oder Eislers »Solidaritätslied « Halt gemacht (dazu im Überblick Juhasz 1994: 14f.). Damit sich nun aber der Begriff der politischen Musik nicht vollends ins Vage auflöse bzw. der Rezipientenwillkür anheim gegeben werde und mithin jede Musik als politische Musik bezeichnet werden könne, fordert Hanns-Werner Heister (1997: Sp. 1666): „Was politisch gemeint ist, muß sich im Gestaltungsprozeß selber objektivieren.« Doch sogleich heißt es einschränkend: Die ästhetische Objektivierung, also die Forderung, dass sich das Politische im Material und in der Musiksprache selbst wiederfinden lasse, »sollte freilich nicht überstrapaziert werden« (ebd.: Sp. 1667). In diesem Sinn durchaus konsequent wird denn auch bei seinen vielen Beispielen zur politischen Musik von der französischen Revolution bis in die Gegenwart auf die Darstellung musikstruktureller Gegebenheiten verzichtet. Und immerhin hatte kein Geringerer als Carl Dahlhaus einschränkend schon zu Beginn der Diskussion um das Politische in Musik darauf verwiesen, dass z.B. autonome Musik zwar keine politische Musik, wohl aber politisch sein könne, da die Decodierung des musikalischen Materials von der ideologischen Haltung der Hörenden abhänge (Dahlhaus 1972: 6). So ist bekanntlich Beethovens 9. Sinfonie, insbesondere der Finalsatz, im Verlauf der bisherigen Rezeptionsgeschichte von so ziemlich allen politischen und weltanschaulich-religiösen Richtungen für ihre Ziele in Anspruch genommen worden (dazu Rummenhöller 1978).

Die in der deutschen Musikwissenschaft bislang geführte Diskussion um politische Musik bzw. das Politische in der Musik bewegt sich also im Kreis und ist - sieht man einmal von Detailanalysen so mancher Kompositionen von Henze, Nono oder Stockhausen ab - wenig erhellend. Auch nicht viel besser stellt sich die Situation in der deutschsprachigen Popularmusikforschung der Nachkriegszeit dar (mit den üblichen, die Regel bestätigenden Ausnahmen: Salzinger 1982; Denselow 1991; Büsser u.a. 2000). Die politische Dimension von volkstümlichem oder deutschem Schlager und überhaupt von Mainstream-Popmusik gar wurde größtenteils getreu dem Adornoschen Ansatz generell als politisch nur ex negativo dargestellt, als Eintauchen in den schönen Schein kulturindustrieller Verdummung (siehe aber Riethmüller 1988; Schoenebeck 1994). Angesichts derartiger Überbautheorien scheint mir die in den 1980er Jahren propagierte Dissidenztheorie von Diedrich Diederichsen doch schon wesentlich differenzierter zu sein. Rock und Pop werden von inm als Katalysator und Motor soziokultureller und politischer Bewegungen verstanden. Vor allem Rockmusik besitze, jenseits ihrer kulturindustriellen Vereinnahmung, eine durchaus ernst zu nehmende, subversive Kraft, oder, mit den Worten von Sarah Thornton (1995: 116ff.): ein beachtliches »subkulturelles Kapital«. Dieses zeige sich in der Negation 
von tradierten Werten (z.B. Rolling Stones: »(I Can't Get No) Satisfaction«), in bissig satirischen Songs (z.B. der Mothers of Invention) oder Soundcollagen (Jimi Hendrix: "Star Spangled Banner «) und in gezielten verbalen Attacken (Bob Dylan: »Masters Of War«). Die musikalisch-textliche Rebellion gegen die Definitionsmacht im jeweils herrschenden politischen System funktioniere nach unterschiedlichen, sich gegenseitig stützenden Aktionsmustern. Die Auflösung bzw. Zerstörung bestehender Wertvorstellungen und Konventionen im gesamtgesellschaftlichen und politischen Bereich erfolge durch Unterwanderung, Kommunikationsverweigerung, Als-Ob-Haltung, Pseudoaffirmation und Geheimsprachenmetaphorik. Die popmusikalischen Strukturen allerdings stellen dabei, so Simon Frith (1983: 15), im Wesentlichen eine Art »black box« dar, in die die zur Entstehungszeit jeweils aktuellen sozialen, ökonomischen, technologischen und kulturell-kommunikativen Variablen des direkten Umfelds symbolhaft eingebunden sind oder aber auch vom Hörer eingebunden werden. Wie das funktionieren kann, hat gerade Alenka Barber-Kersovan (2003) am Beispiel des slowenischen Punks und seinem Beitrag zur Demontage des sozialistischen Wertesystems minutiös beschrieben und ähnlich auch Mathias Lehmann (2003) mit seinen vergleichenden Analysen mehrerer Bühnenwerke in seiner Arbeit Der Dreißigjährige Krieg im Musiktheater während der NS-Zeit.

\section{Modellentwurf zum Beziehungssystem Musik}

Angesichts der Problematik, die sich bei dem Versuch ergibt, den Begriff politische Musik derart mit Inhalt zu füllen, dass er sich nicht ins Vage verflüchtigt, habe ich vor vier Jahren einen ersten Modellentwurf über »politisch engagierte « bzw. »politisierte « Musik zur Diskussion gestellt (Rösing 2000: 315). Entscheidend für diesen Modellentwurf war, dass die gängige Trennung in »reine « Musik und »außermusikalische « Zutaten von mir aufgehoben worden ist. Denn die weit verbreitete Auffassung, Musik bestimme sich allein durch ihre formalen und strukturellen Eigenheiten, sei also ein vornehmlich selbstreferentielles Produkt tönend bewegter Formen, greift meines Erachtens zu kurz. Diese Auffassung wird der kommunikativen Kraft von Musik nicht gerecht. Das Adornosche Bonmot, ein Dreiklang könne weder kapitalistisch noch sozialistisch sein, ist so treffend in Bezug auf das die musikwissenschaftliche Forschung beherrschende Credo einer reinen Musik wie falsch im Hinblick auf die alltägliche Erfahrung, dass das musikalische Produkt immer in einen mehrdimensionalen Zirkulationsprozess eingebunden ist. Die musikimmanente Ebene des musikalischen Produkts 
(die so genannte »reine« Musik) stellt lediglich eine - und nicht zwingend die wichtigste - von mehreren Komponenten (also dem angeblich »Außermusikalischen «) im Beziehungssystem Musik dar.

Der umgangssprachliche Musikbegriff ist vieldimensional. Er kann, ohne dass dies explizit gesagt wird, die musikalische Produktionshandlung, die Vermittlungsschritte bis hin zur klingenden Realisation des musikalischen Produkts, die Rezeption und verschiedene Stadien der Weiterverwertung umfassen. Diese Vieldimensionalität des Musikbegriffs hat in der traditionellen Musikwissenschaft eine Einengung erfahren. Im Gefolge des Autonomiekonzepts des 19. Jahrhunderts wurde der Musikbegriff zum Synonym für das musikalische Produkt. Die 1985 veröffentlichten Statements von Carl Dahlhaus und Hans Heinrich Eggebrecht über die Frage Was ist Musik? machen das sehr deutlich. Alles, was nicht im musikalischen Produkt, also der »reinen « Musik selbst enthalten ist, liegt innen zufolge »außerhalb der Musik«: Der »musikalische Sinn« sei in der Form, im »Formsinn« der Musik gleichsam verdinglicht. Die Interpretation dessen, was hier, also in der musikalischen Struktur, nicht gegenwärtig ist, bezeichnet Eggebrecht deswegen schlichtweg als »riskant« (Dahlhaus/Eggebrecht 1985: 68 u. 139ff.). Carl Dahlhaus knüpft daran - u.a. in direkter Replik auf Zofia Lissa, die in ihren Schriften immer wieder behauptet hatte, das Spezifische von Musik sei gerade ihr nicht spezifisch Musikalisches - abschließend die zentrale Frage, ob der musikalische Gehalt bereits in notierter Musik vorhanden sei oder ob er sich nicht vielmehr erst in der Kommunikation zwischen Hörer und Interpret erschließe (ebd.: 194). Die Beantwortung überlässt er allerdings dem geneigten Leser - er selbst wagt sich dort nicht weiter.

Aus meiner Sicht ist diese Frage eindeutig beantwortbar. Die musikalische Produktionshandlung, die verschiedenen Vermittlungsschritte bis zur klingenden Realisation des musikalischen Produkts und die Rezeption bilden eine unverbrüchliche Einheit. Das erklingende Musikprodukt entfaltet Wirkung immer erst in der Wahrnehmung, sei es im Rahmen einer Live-Darbietung, während der Lautsprecherwiedergabe oder - im Extremfall - sogar beim stumm-imaginativen Lesen von Noten. Darüber hinaus entfaltet sich ihre Wirkung immer in einer bestimmten, wirkungsbeeinflussenden und wirkungskonkretisierenden Situation.

In meinem nun noch einmal überarbeiteten Modellentwurf zum Beziehungssystem Musik und zur politischen Musik (siehe die Grafik auf der folgenden Seite) sind in Rubrik I (»Musik«) sieben der für den musikalischen Zirkulationsprozess und das Kommunikationssystem Musik relevante Stationen aufgeführt. Ihre Abfolge kann im Einzelnen variieren, je nachdem, um welchen Musiktyp es sich handelt (notiertes Werk, improvisierte Musik, im 


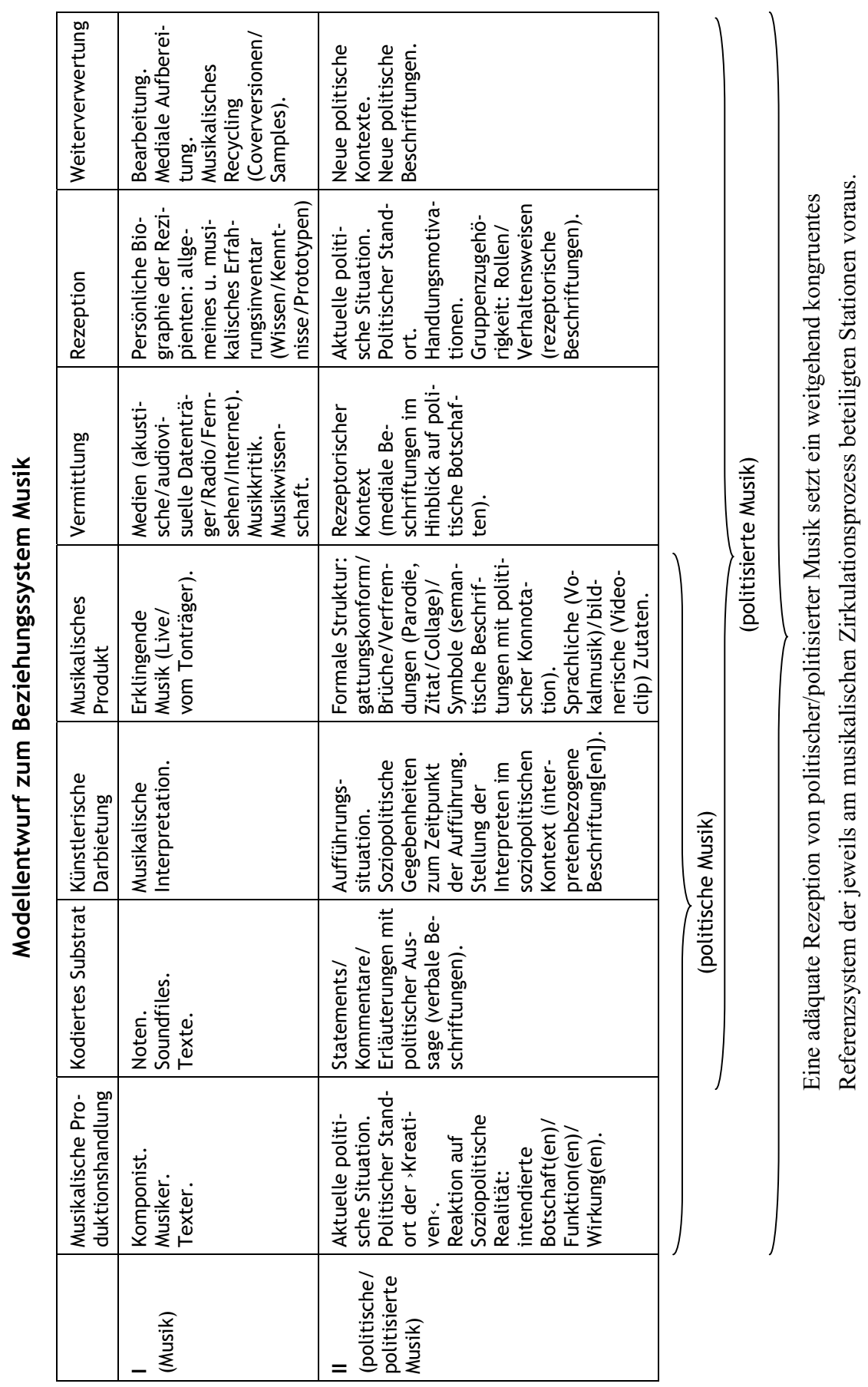


Studio erstellte Musik), wie lange das musikalische Produkt bereits existiert und Bestandteil einer kulturellen Tradition geworden ist (Nationalhymne, tönende Symbole, musikalische Prototypen) und wie häufig es im Verlauf der Zeit bereits den Kreislauf der Neuinterpretation oder Weiterverarbeitung durchgemacht hat.

In Rubrik II habe ich stichwortartig einige jener Komponenten angeführt, die das System Musik mit politischem Gehalt anreichern können: von intendierten politischen Botschaften über so manche, in die musikalische Struktur eingeschriebene Semanteme bis hin zu den vielfältigen Beschriftungsmöglichkeiten verbaler, interpretatorischer, medialer und rezeptorischer Provenienz. Stimmen die politischen Intentionen der musikalischen Produktionshandlung mit den in das kodierte Substrat eingebundenen verbalen und den in der musikalischen Struktur des Produkts enthaltenen semantischen Beschriftungen überein, so handelt es sich dabei um genuin politische Musik. Erfolgt die politische Zuordnung dagegen über interpretenbezogene, mediale bzw. rezeptorische Beschriftungen oder aber durch eine Integration in neue gesellschaftspolitische Kontexte und entsprechende neuen Beschriftungen (z.B. in Form einer ideologischen Vereinnahmung des musikalischen Produkts), dann sollte man von politisierter Musik sprechen. Doch unabhängig davon, ob es sich um genuin politische oder um politisierte Musik handelt, gilt grundsätzlich: Die adäquate Rezeption politischer Botschaften in Musik setzt ein weitgehend kongruentes Referenzsystem aller am musikalischen Zirkulationsprozess beteiligten Stationen voraus.

Ist diese Voraussetzung nicht gegeben, kommt es also nicht zur Deckung von kompositorisch intendierten, im Vermittlungsgeschehen enthaltenen und bei der Rezeption realisierten politischen Funktionen, so sind drei weitere Rezeptionsformen möglich:

1. Das musikalische Produkt ist autonom bzw. ohne politisches Intentionat. Im musikalischen Zirkulationsprozess aber erfolgt entgegen der kompositorischen Absicht eine Aufladung mit politischen Inhalten. So wurde z.B. Liszts sinfonische Dichtung »Les Préludes« allein deswegen zu politisierter Musik, weil ein kurzes Zitat daraus die Rundfunkmeldungen des Wehrmachts-Oberkommandos während des Zweiten Weltkriegs einzuleiten pflegte. Eine neue politische Beschriftung im Zuge der musikalischen Weiterverwertung hat zu einer Umfunktionierung des musikalischen Produkts geführt, die für dessen Rezeption in nationalsozialistischer Zeit und weit darüber hinaus prägend gewesen ist. Oder aber, um ein Beispiel aus der Popmusik anzuführen: Der durchaus harmlose Song »Dancing In The Streets« von Martha Reeves \& The Vandellas (Motown) wurde nach der 
Ermordung von Martin Luther King 1968 kurzerhand zum Schlachtruf für Straßenkämpfe in Detroit umgedeutet.

2. Auch der umgekehrte Fall ist denkbar. Dem Intentionat nach unterliegt politisch-funktionale Musik der Ästhetisierung. So wurde z.B. französische Revolutionsmusik, die ich Teilnehmern eines Seminars über politischengagierte Musik ohne entsprechende Vorab-Information zu Gehör brachte, nach rein formalen und ästhetischen Kriterien gehört, beschrieben und gedeutet. Marschartige Versatzstücke und selbst ein Finale mit Anklängen an die Marseillaise waren als symbolische Codes nicht stark genug, um beim heutigen Hörer in der abstrakten Seminarraum-Situation das politische Anliegen des musikalischen Produkts in den Bewusstseinshorizont der Wahrnehmung zu rücken. Ähnlich auch erging es unlängst der Zuhörerschaft von Liedern, die die tschechische Musiklehrerin Ludmilla Peškařowá während ihrer Gefangenschaft in Ravensbrück als klingende Dokumente der Grausamkeit geschaffen hat. Obwohl die Lieder in der ehemaligen Textilfabrik des Frauenkonzentrationslagers zu hören waren, war ihre emotionale Kraft deutlich stärker als die in den tschechischen Texten mitschwingende und im Programmheft wiedergegebene politische Aussage: Klage, seelische Erschütterung und die Sehnsucht nach Geborgenheit (dazu Knapp 2003: 131ff.) dominierten den Rezeptionsprozess.

3. Das Intentionat der an der musikalischen Produktionshandlung Beteiligten ist unter anderem auch ein politisches. Das musikalische Produkt weist jedoch keine ersichtlichen semantischen Beschriftungen mit politischer Konnotation auf. Dennoch wird ein wie auch immer geartetes politisches Engagement durch zusätzliche Beschriftungen im musikalischen Zirkulationsprozess transportiert, etwa durch entsprechend eindeutige Songtitel, durch Statements der Musikerinnen und Musiker in der Presse, im Internet, auf dem CD-Cover und nicht zuletzt durch die Aufführungssituation selbst (z.B. Benefizkonzert in Anwesenheit von Feuerwehrleuten, Polizisten und freiwilligen Helfern des Terroranschlags in New York).

Mit anderen Worten: Eingriffe in den Zirkulationsprozess des Beziehungssystems Musik können zur nachhaltigen Änderung musikalischer Botschaften führen. In Erläuterungen und Erklärungen zu Musikstücken werden meistens viele der Komponenten, die zum musikalischen Zirkulationsprozess und folglich zum System Musik im ganzheitlichen Sinn gehören, ausgeblendet. Eine derartige Beschränkung auf das musikalische Produkt ist zwar bei musikwissenschaftlichen Analysen üblich, verfehlt aber den zu analysierenden 
Gegenstand. Denn das musikalische Produkt ist, so die lapidare Formulierung von Hans Heinrich Eggebrecht, »ohne Begriffe « (Dahlhaus/Eggebrecht 1985: 192); Begrifflichkeit entsteht erst im wie auch immer gearteten musikalischen Zirkulationsprozess. Musik erweist sich somit als ein soziales System in der Art, wie das Niklas Luhmann (1984: 240) definiert hat: Soziale Systeme bestehen aus »Kommunikation und aus deren Zurechnung als Handlung« (weiterführend dazu Helms 2003 und Phleps 2003).

\section{Fazit}

Die Antwort auf die im Titel gestellte Frage »Wie politisch kann Musik sein? « fällt unterschiedlich aus - je nachdem, was unter dem Begriff Musik verstanden wird.

Bezeichnet man - so mein Vorschlag - den gesamten musikalischen Zirkulationsprozess im Beziehungssystem Musik als Musik, dann kann sie auf sehr vielfältige Weise politisch sein. Allerdings ist die politische Botschaft nicht ein für allemal fixiert. Sie ist veränderbar, und zwar je nachdem, welche Beschriftungen dem musikalischen Produkt im Zirkulationsprozess zugeordnet werden. Genuin politische Musik kann ästhetisiert werden, ihre Botschaft umgemünzt, ihr Engagement für oder gegen eine bestimmte politische Richtung umfunktioniert und ideologisch anderweitig vereinnahmt werden. Und ebenso kann natürlich ein der Intention nach erst einmal unpolitisches Musikprodukt je nach den speziellen Gegebenheiten im musikalischen Zirkulationsprozess politisiert werden.

Daraus folgt, dass das musikalische Produkt selbst, in der herkömmlichen Terminologie die »reine « Musik, keine konkrete Botschaft enthält, also weder politisch noch männlich oder religiös usw. ist. Es handelt sich hier um ein Regelwerk von Tönen, Klängen und Zusammenklängen, Rhythmen, Motiven, Melodien u.a.m., die ihren Sinn in sich selbst tragen und gemäß den jeweils gültigen Maximen des Musikmachens funktionieren.

Man mag die darin zum Ausdruck kommende Begriffslosigkeit musikalischer Produkte bedauern. Man kann diese Begriffslosigkeit aber auch als ihre besondere Stärke und als entscheidenden Leistungsvorsprung z.B. gegenüber der Sprache begreifen. Denn das musikalische Produkt hat dank seiner Nicht-Diskursivität ein erhebliches Transzendenzpotential. Es bietet sich darum - in Verbindung mit den vielen zusätzlichen Beschriftungsmöglichkeiten von der musikalischen Produktion bis hin zur Rezeption - als Projektionsfläche für Emotionen, Imaginationen und Assoziationen an. Jede musikbezogene Symbolbildung und Bedeutungszuweisung vollzieht sich mit- 
hin im mehrdimensionalen Feld des gesamten Beziehungssystems Musik. In jeder Analyse und Interpretation von Musik in diesem ganzheitlichen Sinn müssen die einzelnen Variablen des Zirkulationsprozesses so exakt wie möglich beschrieben und ihre Interaktionen offen gelegt werden, um zugleich auch deutlich werden zu lassen, dass jede Bedeutungszuweisung wiederum in dem Maß revidierbar ist, in dem sich die Beschriftungsbedingungen ändern oder bereits geändert haben.

\section{Epilog}

Der von mir hier zur Diskussion gestellte Modellentwurf zum System Musik ist weder neu noch originell. Er beschreibt Selbstverständlichkeiten, die im (wissenschaftlichen) Umgang mit Musik allerdings in dem Maß abhanden gekommen sind, in dem das musikalische Produkt im Gefolge der Autonomieästhetik des 19. Jahrhunderts in den Focus der Aufmerksamkeit gelangt ist. In Umkehrung des Spruchs zum 11. September - »Nichts wird mehr so sein, wie es war« - muss es also eher heißen: Alles bleibt, wie es war. Das betrifft nicht nur die Musik und ihre vielfältigen, zum Teil eben auch politischen Funktionen in der Gesellschaft, sondern so gut wie alle Bereiche des öffentlichen und privaten Lebens. Art Spiegelman hat das in seiner zehnteiligen Cartoon-Serie »Im Schatten keiner Türme« mit Replik auf Leonardo da Vincis »Abendmahl« und auf apokalyptische Weltuntergangsvisionen u.a. liebevoll-ironisch am Beispiel eines letztlich höchst profanen Handtaschenraubs dargestellt (Kommentar der betroffenen Person: »Ich war soooo erleichtert! ... Endlich wird es wieder so sein wie früher! «; siehe Die Zeit, Nr. 34 v. 14. August 2003, S. 18), die Tageszeitung Bild in ihrer Ausgabe "zwei Jahre nach dem Schrecken « am Beispiel eines persönlichen Schicksals gemäß dem Motto »Lebensmut besiegt das Böse« (Bild v. 11. September 2003, S. 12). 


\section{Literatur}

Adorno, Theodor W. (1962). Einleitung in die Musiksoziologie. Zwölf theoretische Vorlesungen. Frankfurt/M.: Suhrkamp.

Barber-Kersovan, Alenka (2003). Vom »Punkfrühling« zum »Slowenischen Frühling«. Der Beitrag des slowenischen Punk zur Demontage des sozialistischen Wertsystems. Hamburg: Phil. Diss. (Musikwissenschaft).

Brown, Damon (2002). »Masterminds :: Stone Soup :: Third Earth Music. In: RapReviews, 29. März [http://www.rapreviews.com/archive/2002_03_stonesoup. html, Zugriff: 24.3.2004].

Büsser, Martin, u.a. (Hg.) (2000). Pop und Krieg (= testcard - Beiträge zur Popgeschichte 9). Mainz: Ventil Verlag.

Dahlhaus, Carl (1972). »Thesen über engagierte Musik.«In: Neue Zeitschrift für Musik 133, H. 1, S. 3-8.

Dahlhaus, Carl / Eggebrecht, Hans Heinrich (1985). Was ist Musik? Wilhelmshaven: Noetzel.

Denselow, Robin (1991). The Beat Goes On. Popmusik und Politik: Geschichte einer Hoffnung. Reinbek: Rowohlt.

Diederichsen, Diedrich (Hg.) (1993). Yo! Hermeneutics! Schwarze Kulturkritik. Pop, Medien, Feminismus. Berlin: Edition ID-Archiv.

Flammer, Ernst H. (1981). Politisch engagierte Musik als kompositorisches Problem, dargestellt am Beispiel von Luigi Nono und Hanns Werner Henze. BadenBaden: Körner.

Frith, Simon (1983). Sound Effects. Youth, Leisure and Politics of Rock'n'Roll. London: Constable.

Goebbels, Heiner (1980). »Rock gegen Rechts - Ein Mißverständnis?« In: Rock gegen Rechts. Musik als politisches Instrument. Hg. v. Bernd Leukert. Frankfurt/M.: Fischer, S. 87-96.

Heister, Hanns-Werner (1997). »Politische Musik. « [mit ausführlichen Literaturhinweisen] In: Musik in Geschichte und Gegenwart. Sachteil Bd. 7. Hg. v. Ludwig Finscher. Kassel, Stuttgart: Bärenreiter/Metzler (2. Aufl.), Sp. 1661-1682.

Helms, Dietrich (2003). "Auf der Suche nach einem neuen Paradigma: Vom System Ton zum System Sound. «In: Pop Sounds. Klangtexturen in der Pop- und Rockmusik. Basics -Stories - Tracks. Hg. v. Thomas Phleps u. Ralf von Appen (= texte zur populären musik 1). Bielefeld: transcript, S. 197-228.

Juhasz, Christiane (1994). Kritische Lieder und Politrock in Österreich. Eine analytische Studie. Frankfurt/M. u.a.: Peter Lang.

Knapp, Gabriele (2003). Frauenstimmen. Musikerinnen erinnern sich an Ravensbrück. Berlin: Metropol.

Kneif, Tibor (1977). Politische Musik? (= Fragmente als Beiträge zur Musiksoziologie 6). Wien: Doblinger.

Kolleritsch, Otto (1972). »Musik zwischen Engagement und Kunst. In: Musik zwischen Engagement und Kunst. Hg. v. Otto Kolleritsch (= Studien zur Wertungsforschung 3). Wien: Universal Edition, S. 92-99.

Lehmann, Mathias (2003). Der Dreißigjährige Krieg im Musiktheater während der NS-Zeit. Hamburg: Phil. Diss. (Musikwissenschaft).

Niklas Luhmann (1984). Soziale Systeme. Grundriß einer allgemeinen Theorie. Frankfurt/M.: Suhrkamp. 
Pauli, Hansjörg (1981). »Politische Musik.«In: Funkkolleg Musik. Bd. 2. Hg. v. Carl Dahlhaus. Frankfurt/M.: Fischer, S. 369-387.

Phleps, Thomas (1993). »Musik und Ideologie. «In: Musikpsychologie. Ein Handbuch. Hg. v. Herbert Bruhn, Rolf Oerter u. Helmut Rösing. Reinbek: Rowohlt, S. 94103.

Phleps, Thomas (2003). »Pop sounds so und Pop Sound so und so. Einige Nachbemerkungen vorweg. "In: Pop Sounds. Klangtexturen in der Pop- und Rockmusik. Basics -Stories - Tracks. Hg. v. Thomas Phleps u. Ralf von Appen (= texte zur populären musik 1). Bielefeld: transcript, S. 11-18.

Riethmüller, Albrecht (1988). »Zur Politik der unpolitischen Musik.«In: Funkkolleg Musikgeschichte. Europäische Musik vom 12.-20. Jahrhundert. Studienbegleitbrief 11. Hg. v. Deutschen Institut für Fernstudien an der Universität Tübingen. Weinheim, Basel, Mainz: Beltz/Schott, S. 11-41.

Rösing, Helmut (2000). "Kann Musik politisch sein? Zur Rolle der Musik in Stanley Kubricks Film 'Full Metal Jacket‘.«In: Komposition als Kommunikation. Zur Musik des 20. Jahrhunderts. Hg. v. Constantin Floros (= Hamburger Jahrbuch für Musikwissenschaft 17). Frankfurt/M. u.a.: Peter Lang, S. 313-322.

Rösing, Helmut (2002). »Männlichkeitssymbole in der Musik - eine Spurenlese.« In: Festschrift Ekkehard Jost zum 65. Geburtstag. Hg. v. Bernd Hoffmann, Franz Kerschbaumer, Franz Krieger u. Thomas Phleps (= Jazzforschung / Jazz Research 34). Graz: Akademische Druck- und Verlagsanstalt, S. 243-256.

Rösing, Helmut (2003). »Pop in der Kirche? Überlegungen zur Funktion von populärer Musik in der Erlebnisgesellschaft. «In: Popularmusik und Kirche - Positionen, Ansprüche, Widersprüche. Hg. v. Wolfgang Kabus. Frankfurt/M. u.a.: Peter Lang, S. 33-51.

Rummenhöller, Peter (1978). Einführung in die Musiksoziologie. Wilhelmshaven: Heinrichshofen.

Salzinger, Helmut (1982). Rock Power oder Wie musikalisch ist die Revolution? Reinbek: Rowohlt.

Schoenebeck, Mechthild von (1994). »'Wenn die Heidschnucken sich in die Äuglein gucken...<. Politische Inhalte des volkstümlichen Schlagers.«In: Beiträge zur Popularmusikforschung 13, S. 6-24.

Schoenebeck, Mechtild von (1987). Was macht Musik populär? Untersuchungen zu Theorie und Geschichte populärer Musik. Frankfurt/M. u.a.: Peter Lang.

Thornton, Sarah (1995). Club Cultures: Music, Media and Subcultural Capital. Cambridge: Polity Press.

\section{Abstract}

An inspection of all the popular music productions which were concerned explicitly with the terrorist plot of the September 11th in 2001, is leading to the crucial question: Can music be political - without further explanation or text? The musical product, the spure music surely can not. But the musical system of relations, including the process of circulation in total that means the production, distribution and reception of music, may be political. During the process of circulation music will be enriched with meaning. But this meaning is not finally fixed. It may change in dependence on new or other facts due to the process of music circulation. 